\title{
The Relationship between Academic/Professional Qualification and Longevity of Service Parameters in Trans-Cultural Client Care by Nurses of Fako, SWR, Cameroon
}

\author{
Mary Bi Suh Atanga1, Roland Acha Achi², John Ngunde Palle ${ }^{2}$ \\ ${ }^{1}$ Department of Nursing/Midwifery, Faculty of Health Sciences, University of Bamenda, Cameroon \\ ${ }^{2}$ Department of Nursing, Faculty of Health Sciences, University of Buea, Cameroon \\ Email: *mbisuh@yahoo.com
}

Received 19 February 2016; accepted 5 March 2016; published 10 March 2016

Copyright (C) 2016 by authors and OALib.

This work is licensed under the Creative Commons Attribution International License (CC BY). http://creativecommons.org/licenses/by/4.0/

(c) (i) Open Access

\section{Abstract}

Every client seeking care comes from a cultural background. Each cultural background has elements that need to be diagnosed in order to provide care in a manner that is seriously conflicting with the client's culture. Sometimes in the provision of care, conflicting ideas need negotiation and sometimes re-patterning, but these must be done in an acceptable manner such that the client is at ease, and so healing is enhanced. For these to occur, knowledge and qualification of the care provider play a role. At other times, the longevity of service and knowledge and attitudes either interfere or enhance the process. Based on these, this study on the relationship between academic/ professional qualification and longevity of service was conducted to test the presence or non presence of trans-cultural client care among some nurses and midwives in Health Care Institutions in Fako Division. The objectives were to sought the importance and preservation of transcultural client care (TCC); find out the relationship between academic/professional qualification; and relationship between longevity of service and the identified parameters of culture diagnostic, negotiating and re-patterning. Stratified samples of nurses/midwives working in Health Care Institutions in Fako Division, were administered multiple choice questionnaires, for a period of three weeks. One hundred and sixty five (165) participants filled the questionnaires and SPSS 16.0 was used to analyze the data collected. The results revealed high degree of dependence between the professional qualification $(\mathrm{P}$-value $=\mathbf{0 . 0 2 3})$, academic qualification $(\mathrm{P}$-value $\mathbf{0 . 0 0 1})$, professional profile (longevity) $(\mathrm{P}$-value $=0.000)$ of nurses $/$ midwives and trans-cultural client care in healthcare institutions in Fako Division. These verifications let to the confirmation of the assumptions of the study. Professional qualification/academic qualification, and professional profile

\footnotetext{
${ }^{*}$ Corresponding author.
}

How to cite this paper: Atanga, M.B.S., Achi, R.A. and Palle, J.N. (2016) The Relationship between Academic/Professional Qualification and Longevity of Service Parameters in Trans-Cultural Client Care by Nurses of Fako, SWR, Cameroon. Open Access Library Journal, 3: e2278. http://dx.doi.org/10.4236/oalib.1102278 
(longevity), influence trans-cultural client care among nurses/midwives in health institutions in Fako Division. We recommend that Health Care Institutions should regularly organize seminars and workshops on the effective use and application of clients' culture during care.

\title{
Keywords
}

Trans-Cultural Client Care, Academic/Professional Qualification, Longevity, Importance, Preservation, Relationships with Culture Diagnostics, Negotiating, Re-Patterning

\author{
Subject Areas: Nursing
}

\section{Introduction}

Nurses and midwives need or require adequate theoretical knowledge and skills to permit them to provide culturally congruent (competent) care to clients. Examples of such body of knowledge and skills include; medical sociology and anthropology, multidisciplinary counseling, culture care assessment, trans-cultural communication, culture desire, cultural awareness, cultural knowledge, cultural sensitivity and cultural competence [1]. The variation of knowledge and skills acquired by the different categories of nurses and midwives through training and field experience might influence their ability to provide trans-cultural care to clients.

Until recently, the idea of curing and not healing was the main interest in the Western mode of health care with more emphases was laid on the technology, power, analysis and repair of damaged parts. This notion placed the cure giver at a superior and active position and the care receiver in a somewhat lower and passive position. This was mainly due to the fact that, healing was largely overlooked and far way less understood than the pathophysiology of disease.

Medicine and/or health care is in the $3^{\text {rd }}$ era of evolution wherein the physical aspects of health care is rather a small portion relatively very insufficient or perhaps obsolete in its entirety to be considered complete relative to required standards. Health care delivery systems and providers must possess facilities and competence sufficient enough to provide healthcare that goes beyond just the physical aspects as was the case in the 1860s [2].

Era III emphasizes the fact that, the quality of interaction between individuals (giver/sender and receiver) determines and influences healing. In this case the sender is the healthcare provider while the receiver is the client. This underlines a phenomenon of human interaction, which goes beyond the physical or tangible aspects. Thus requiring cultural, spiritual and other non-physical attributes as preconditions for a start [2].

Several healing modalities and alternative medical therapies exist but vary a great deal between cultures [3]. These modalities and alternatives can be integrated into client care to foster rapid and total healing. Nurses and midwives need be culturally competent in order to determine a client's cultural identity and preferences and consequently adapt client care to be culture specific and universal. The variation in cultural competence among them may equally determine and perhaps influence their performance as far as trans-cultural client care is concerned.

Every human being belongs to a culture which has its specific ways of handling health related issues. Therefore, clients' wellbeing would be enhanced if culturally-based and beneficial care services are provided to them. This will prevent and reduce stress, ethical and moral problems, cultural conflicts and non-compliance [4].

Sager [5], supported this idea by emphasizing that the knowledge in trans-cultural nursing (TCN) is an inevitable resource in current day health care practice. The prove of this notion in health care can be noticed in the application of trans-cultural knowledge in a broad scope such as in nursing curricula and health care practice guidelines.

The central idea of the Theory of Human Caring is that "humans cannot be treated as objects, and that humans cannot be separated from self, other, nature, and the larger universe" The theory focuses on "the centrality of human caring and on the caring-to-caring transpersonal relationship and its healing potential for both the one who is caring and the one who is being cared for" [6].

In Watson's view, the disease might be cured, but illness would remain because without caring, health is not attained. Caring is the essence of nursing and connotes responsiveness between the nurse and the person; the 
nurse co-participates with the person. Watson contends that caring can assist the person to gain control, become knowledgeable, and promote health changes.

Nursing is generally offered as an associate's or bachelor's degree program. Even though the traditional focus of nursing was to teach the knowledge and skills to enable the nurses to practice in the hospital setting, nursing now respond to new scientific knowledge and technology, cultural, political and socioeconomic changes in society such that nursing curricula are continually being revised to meet the needs of nurses working in changing environment. Programs of nursing are increasingly based on a broad knowledge of biologic, social and physical sciences as well as liberal and humanities.

Nursing research entails developing and expanding knowledge about human responses to actual or potential health problems and investigating the effects of nursing actions on those responses. The major goal of nursing research is to improve client care and trans-cultural client care is one aspect that must be emphasized. In it, nurses depict cultural competence as having the ability to understand cultural differences in order to provide quality care to a diversity of people. Culturally competent nurses are sensitive to issues related to culture, race, ethnicity, gender, and sexual orientation. Furthermore, culturally competent nurses have achieved efficacy in communication skills, cultural assessments, and knowledge acquisition related to health practices of different cultures. Cultural competence involves nurses continuously striving to provide effective care within the cultural confines of their patients [4] [7]-[9]. There are several methods through which culturally competent care can be rendered, with each method or procedure having specific issues to identify and handle.

\subsection{Statement of Problem}

Health care delivery nowadays is greatly influenced by theories, concepts and principles which constantly change in respond to clients' needs, new research findings and technological know-how. The recipients or consumers of health care services often provide complex and ever changing healthcare demands consequent to socio-cultural and environmental changes. The tasks and responsibilities of health care providers are definitely very challenging and complex. The ideal standard warrants a prompt and dynamic respond through harmonization of new research and technological findings with actual client needs [2].

The quality of care given to clients in health care institutions on the other hand may vary based on general and professional educational background, working experience and in-service training of nurses and midwives. New issues like respect for clients' culture that plays an important role in the healing process may still be neglected by some nurses and midwives. This is either due to less knowledge or poor attitudes, or just the lack of knowledge on trans-cultural client care. There appear however to be a relationship between academic/professional qualification, and longevity of service to parameters of trans-cultural client care like culture diagnostic, negotiating and re-patterning. This relationship is yet to known, hence, the reason why the research was undertaken.

\subsection{Research Questions}

1) What is importance and preservation of trans-cultural client care among nurses and midwives of Fako division, South West Region, Cameroon?

2) What is the relationship between academic/professional qualification and knowledge/attitudes of culture diagnostic, negotiating and re-patterning in trans-cultural client care among these health care providers?

3) What is the relationship between longevity of service and knowledge/attitude of culture diagnostic, negotiating and re-patterning in trans-cultural client care among these health care providers?

\subsection{Assumptions}

1) The academic/professional qualification of nurses and midwives influences trans-cultural client care in health care institutions in Fako Division.

2) The professional profile (longevity of service and in-service training) of nurses and midwives influences trans-cultural client care in health care institutions in Fako Division.

\subsection{Objectives}

1) To assess the importance and preservation of trans-cultural client care among nurses and midwives of Fako division, South West Region, Cameroon. 
2) To assess the relationship between academic/professional qualification and knowledge/attitudes of culture diagnostic, negotiating and re-patterning in trans-cultural client care among these health care providers.

3) To assess the relationship between longevity of service and knowledge/attitude of culture diagnostic, negotiating and re-patterning in trans-cultural client care among these health care providers?

\section{Research Materials and Methods}

Based on the research questions and objectives, the research used a cross-sectional descriptive and analytic study design, carried out in Fako Division of the South West Region of Cameroon with many health care institutions among which are 2 referral or Regional hospitals (Buea and Limbe Regional Hospitals). All the health institutions found within the area were eligible for the study but were selected on the basis of stratified sampling that considered all the 223 nurses in the public health care institutions. Health care institutions have a cross section of health personnel of different educational and training background, experience and category. These include, Nursing Aids, State registered nurses, midwives, senior nurses and specialized nurses who needed to be sampled following their strata.

Hence, the stratified sampling technique was used and the target population was stratified into the different nursing categories i.e. senior nurses, higher nursing diploma, state registered, specialized nurses and nurse Aids. Thereafter, proportionate sample of each category were determined. Based on this, Health care Institutions within the study were chosen and the various proportions of the different categories were chosen randomly and administered the research questionnaires and so a representative sample was obtained which in turn strengthened the validity of inferences for generalizations to be made.

This sample size calculation was based on the estimation of the proportion of nurses and midwives found in health institution in Fako Division.

From the number Nurses and midwives in Fako, the minimum sample size needed was calculated using the following formula adopted from Babbie E. [10], Michael and Michael [11], lagers and Justo [12] and Eng [13] as follows:

$$
n=\frac{\left(z^{2}\right) p q}{d^{2}}
$$

where

$n_{o}=$ minimum sample size required for infinite population

$p=$ pre-study estimate of the proportion of personnel in Health institutions in Fako division (and since there is no pre-studied estimate from previous studies, $50 \%$ or 0.5 will be used)

$q=1-p$

$d=$ the degree of precision or the accuracy ( $=5 \%$ or 0.05$)$

$z=$ standard normal variant at confidence level of $95 \%$ (normal value is 1.96 )

Hence,

Sample size $\left(\mathrm{n}_{\mathrm{o}}\right)=(1.96)^{2}(0.5)(0.5) /(0.05)^{2}$

$=0.9604 / 0.0025=385$ persons $/$ participants expected.

But, assuming that the total population of nurses/midwives, $\mathrm{N}$ is about 400 .

The minimum sample size required for the finite population, $n$, is gotten by applying the formula below;

$$
n=\frac{n_{o}}{1+\frac{n_{o}}{N}}
$$

$$
\begin{aligned}
\mathrm{n} & =385 /(1+385 / 400) \\
& =385 / 1.9625 \\
& =\mathbf{1 9 6} \text { (approximately 200) nurses/midwives }
\end{aligned}
$$

Thus the study targeted about 200 nurses/midwifes.

Since a stratified sampling method was used, a proportion of 50\% were sampled (selected) from both the private and the public health care institutions. That is;

- Private health care institutions: $(50 \%) \times(250)=125$ nurses/midwifes

- Public health care institutions: $(50 \%) \times(150)=75$ nurses/midwives 
There, 200 nurses and midwives were to participate in the study in the proportion of 125 from the private and 75 from the Public health care institutions.

However, during the study, 165 nurses/midwives actually participated in the proportion of 100 from private and 65 from public health care institutions in Fako Division.

Data of the study was collected using a questionnaire, comprised of seventeen (17) multiple choice questions divided into sections in accordance with the research objectives.

Apart from the different sections and questions that made up the body of the questionnaire, there was also an introductory section to orientate and facilitate participants' comprehension of the purpose of the study in order to have their consent. The sections and all the questions of the questionnaire each had a theoretical reference. Each of the sections had specific issues (knowledge, skill and attitude) about trans-cultural patient care to measure. The questionnaire was structured such that, it had a key to facilitate and ensure accurate decoding and analysis of the information filled in by the respondents. For validity of this instrument, a pre-test administration was done which assisted in the identification and modification of sections and questions that were difficult, ambiguous and/or difficult to comprehend. The pre-test site was about 50 kilometers away from the study area and used a few nurses

Data was collected by allowing the participants to fill out the questionnaires and handed in on completion. However participants who preferred to fill the questionnaire some other time deemed more convenient than the time of administration of the questionnaire were given a period of at most 3 days to fill in and submit their questionnaires. The respondents were not under pressure and were sources during break or leisure periods while at work.

Questionnaire keys were established and used to facilitate decoding, interpretation and filling of the information into the computer for subsequent analysis. Descriptive statistics was used to describe the relationships between knowledge and attitudes with regards to the issues to be addressed as on the objectives with reference to trans-cultural client care presents and their variations among nurses and midwives in health care institutions Fako Division. Thus, inferential statistics was used to determine the relationship that exists between the studied variables. The data collected were presented using simple percentages before using cross tables in order to analyse and/or investigate the relationship that existed between these variables. Finally tables of statistical test done to verify relationship between variables were presented. For ethical issues to be addressed, participant filled a consent form assuring their informed consent to participate in the study, verbal consent was obtained from participants prior to administration of questionnaire, and none of the participants were obliged to participate in the study. Those who did not consent were persuaded further to consent and otherwise excluded from the study, participants were fully informed of their right to withdraw or retrieve consent any time deemed necessary.

\section{Results}

It can be observed on Table 1 that the number of participants who were of the opinion that patient culture is a very important aspect to be considered during patient care increases as academic qualification increases while those who have no idea on the importance of this aspect, decreases as academic qualification increases.

Table 2 reveals that, the proportion of participants who possess exact knowledge and attitude relative to cultural preservation followed a uniform ascending format or trend with professional qualification, while the proportion of those who were totally ignorant about cultural preservation, decreases uniformly following professional qualification.

Table 3 reveals that, those who were of the opinion that the ethnicity of a client is a good cultural diagnostic determinant, increases as academic qualification increases and those who did not have any idea on this aspect decreases as academic qualification increases. Equally, the choice of tribe as a good diagnostic determinant decreases as academic qualification increases.

Table 4 indicates that, the proportion of participants with exact knowledge and attitude on cultural negotiation during patient care increases following an increase in professional qualification while those with approximate knowledge decrease as professional qualification increase. In the same way, the proportion of those who are totally ignorant about this aspect was high at the lowest professional category.

Table 5 shows that, as professional qualification gets higher, the proportion of participants who possess exact knowledge/attitude relative to cultural re-patterning during patient care increases as well.

Table 6 shows that, the proportion of those who choose tribe as cultural diagnostic determinant increases as longevity of service increases. Likewise, total ignorance on this aspect increases as longevity decreases. 
Table 1. Relationship between academic qualification and opinions on the importance of culture in care.

\begin{tabular}{ccccccc}
\hline \multirow{2}{*}{ Academic Qualification } & \multicolumn{5}{c}{ Patients' Culture in Care } & Total \\
\cline { 2 - 6 } & $\begin{array}{c}\text { Very } \\
\text { Important }\end{array}$ & Important & Sometimes Important & Not Necessary at All & No Idea & \\
\hline Ordinary Level & $51.2 \%$ & $27.9 \%$ & $16.3 \%$ & $2.3 \%$ & $2.3 \%$ & $100.0 \%$ \\
Advance Level & $53.8 \%$ & $24.7 \%$ & $15.1 \%$ & $4.3 \%$ & $2.2 \%$ & $100.0 \%$ \\
Bachelor's Degree & $65.4 \%$ & $23.1 \%$ & $11.5 \%$ & $0.0 \%$ & $0.0 \%$ & $100.0 \%$ \\
Master's Degree & $66.7 \%$ & $33.3 \%$ & $0.0 \%$ & $0.0 \%$ & $0.0 \%$ & $100.0 \%$ \\
Total & $\mathbf{5 5 . 2 \%}$ & $\mathbf{2 5 . 5 \%}$ & $\mathbf{1 4 . 5 \%}$ & $\mathbf{3 . 0 \%}$ & $\mathbf{1 . 8 \%}$ & $\mathbf{1 0 0 . 0} \%$ \\
\hline
\end{tabular}

Table 2. Relationship between professional qualification and knowledge and attitude relative to Cultural preservation during trans-cultural client care.

\begin{tabular}{|c|c|c|c|c|}
\hline \multirow{2}{*}{ Professional Qualification } & \multicolumn{3}{|c|}{ Cultural Preservation } & \multirow{2}{*}{ Total } \\
\hline & Exact & Approximate & Total Ignorance & \\
\hline Nurse Aid & $38.0 \%$ & $46.0 \%$ & $16.0 \%$ & $100.0 \%$ \\
\hline State Registered Nurse & $44.4 \%$ & $51.1 \%$ & $4.4 \%$ & $100.0 \%$ \\
\hline Mid Wife & $50.0 \%$ & $41.7 \%$ & $8.3 \%$ & $100.0 \%$ \\
\hline Senior Nurse & $70.0 \%$ & $26.7 \%$ & $3.3 \%$ & $100.0 \%$ \\
\hline Specialist Nurse & $75.0 \%$ & $25.0 \%$ & $0.0 \%$ & $100.0 \%$ \\
\hline Other (Specify) & $45.8 \%$ & $45.8 \%$ & $8.3 \%$ & $100.0 \%$ \\
\hline Total & $48.5 \%$ & $43.0 \%$ & $8.5 \%$ & $100.0 \%$ \\
\hline
\end{tabular}

Table 3. Relationship between academic qualification and cultural diagnostic determinant.

\begin{tabular}{|c|c|c|c|c|c|c|}
\hline \multirow{2}{*}{ Academic Qualification } & \multicolumn{5}{|c|}{ Diagnostic Determinant } & \multirow{2}{*}{ Total } \\
\hline & Tribe & Clan & Ethnic Group & Race & No Idea & \\
\hline Ordinary level & $79.1 \%$ & $0.0 \%$ & $14.0 \%$ & $4.7 \%$ & $2.3 \%$ & $100.0 \%$ \\
\hline Advance level & $71.0 \%$ & $6.5 \%$ & $14.0 \%$ & $1.1 \%$ & $7.5 \%$ & $100.0 \%$ \\
\hline Bachelor's Degree & $65.4 \%$ & $3.8 \%$ & $23.1 \%$ & $3.8 \%$ & $3.8 \%$ & $100.0 \%$ \\
\hline Master's Degree & $33.3 \%$ & $33.3 \%$ & $33.3 \%$ & $0.0 \%$ & $0.0 \%$ & $100.0 \%$ \\
\hline Total & $71.5 \%$ & $4.8 \%$ & $15.8 \%$ & $2.4 \%$ & $5.5 \%$ & $100.0 \%$ \\
\hline
\end{tabular}

Table 4. Relationship between professional qualification and knowledge/attitude relative cultural negotiation during transcultural client care.

\begin{tabular}{|c|c|c|c|c|}
\hline \multirow{2}{*}{ Professional Qualification } & \multicolumn{3}{|c|}{ Cultural Negotiation } & \multirow{2}{*}{ Total } \\
\hline & Exact & Approximate & Total Ignorance & \\
\hline Nurse Aid & $48.0 \%$ & $46.0 \%$ & $6.0 \%$ & $100.0 \%$ \\
\hline State Registered Nurse & $68.9 \%$ & $31.1 \%$ & $0.0 \%$ & $100.0 \%$ \\
\hline Mid Wife & $75.0 \%$ & $25.0 \%$ & $0.0 \%$ & $100.0 \%$ \\
\hline Senior Nurse & $53.3 \%$ & $46.7 \%$ & $0.0 \%$ & $100.0 \%$ \\
\hline Specialist Nurse & $75.0 \%$ & $25.0 \%$ & $0.0 \%$ & $100.0 \%$ \\
\hline Other (Specify) & $50.0 \%$ & $41.7 \%$ & $8.3 \%$ & $100.0 \%$ \\
\hline Total & $57.6 \%$ & $39.4 \%$ & $3.0 \%$ & $100.0 \%$ \\
\hline
\end{tabular}


Table 5. Relationship between professional qualification and knowledge and attitude relative to cultural re-patterning during trans-cultural client care.

\begin{tabular}{ccccc}
\hline & \multicolumn{3}{c}{ Cultural Re-Patterning } & \multirow{2}{*}{ Total } \\
\cline { 2 - 4 } Professional Qualification & Exact & Approximate & Total Ignorance & $100.0 \%$ \\
Nurse Aid & $48.0 \%$ & $50.0 \%$ & $2.0 \%$ & $100.0 \%$ \\
State Registered Nurse & $53.3 \%$ & $37.8 \%$ & $8.9 \%$ & $100.0 \%$ \\
Mid Wife & $58.3 \%$ & $33.3 \%$ & $8.3 \%$ & $100.0 \%$ \\
Senior Nurse & $60.0 \%$ & $33.3 \%$ & $0.7 \%$ & $100.0 \%$ \\
Specialist Nurse & $75.0 \%$ & $25.0 \%$ & $12.5 \%$ & $100.0 \%$ \\
Other (Specify) & $50.0 \%$ & $37.5 \%$ & $\mathbf{6 . 7 \%}$ & $\mathbf{1 0 0 . 0 \%}$ \\
\hline Total & $\mathbf{5 3 . 3 \%}$ & $\mathbf{4 0 . 0 \%}$ & & \\
\hline
\end{tabular}

Table 6. Relationship between longevity of service and knowledge on client's culture diagnostic determinants during transcultural client care.

\begin{tabular}{|c|c|c|c|c|c|c|}
\hline \multirow{2}{*}{ Longevity of Service } & \multicolumn{5}{|c|}{ Diagnostic Determinant } & \multirow{2}{*}{ Total } \\
\hline & Tribe & Clan & Ethnic Group & Race & No Idea & \\
\hline 0 - 5 Years & $64.6 \%$ & $4.0 \%$ & $21.2 \%$ & $3.0 \%$ & $7.1 \%$ & $100.0 \%$ \\
\hline $6-11$ Years & $76.2 \%$ & $9.5 \%$ & $9.5 \%$ & $0.0 \%$ & $4.8 \%$ & $100.0 \%$ \\
\hline 12 - 17 Years & $87.5 \%$ & $0.0 \%$ & $6.2 \%$ & $6.2 \%$ & $0.0 \%$ & $100.0 \%$ \\
\hline More than 18 Years & $100.0 \%$ & $0.0 \%$ & $0.0 \%$ & $0.0 \%$ & $0.0 \%$ & $100.0 \%$ \\
\hline Total & $71.5 \%$ & $4.8 \%$ & $15.8 \%$ & $2.4 \%$ & $5.5 \%$ & $100.0 \%$ \\
\hline
\end{tabular}

Table 7 indicates that, exact knowledge and attitude on cultural negotiation during client care increases with increase in longevity of service.

Table 8 equally reveals that, exact knowledge/attitude on cultural re-patterning during patient care increases with increase in longevity of service, while total ignorance on this aspect increases as longevity decreases.

\section{Discussion}

It is always common place to understand and believe that higher academic and professional qualifications and longevity of service enables a health care provider to provide care in a better manner than the less ones. This study has demonstrated that the recruitment of less qualified staff does no good to health care provision.

In trans-cultural care, nurses and midwives must be knowledgeable enough to know that people come to hospital with their various cultures. They will be comfortable when the health provider can ask them questions to diagnose their cultures. Negotiate with them on any form of minor re-patterning to assist them get well soon and maintain health.

Longevity of service also plays an important role in making one gain experience into peoples' cultures, but this longevity must go with work consciousness, knowledge and better attitudes. These have been better explained by the cross tables. For example:

\section{Professional Qualification vis-à-vis Trans-Cultural Client Care}

During data analysis, several cross tables were made in order further investigate and ascertain the relationship between professional qualification and trans-cultural client. It was also noticed that, the exact knowledge and attitude increased with professional qualification which gets higher from Nurse Aid up to specialized nurses. This finding is consistent to that of Cliffnotes [14] which supported and insisted on the point that training (be it on-the job, off-the job or both) is an important aspect of employee development and output. 
Table 7. Relationship between longevity at service and knowledge and attitude relative to cultural negotiation during transcultural client care.

\begin{tabular}{|c|c|c|c|c|}
\hline \multirow{2}{*}{ Longevity at Service } & \multicolumn{3}{|c|}{ Cultural Negotiation } & \multirow{2}{*}{ Total } \\
\hline & Exact & Approximate & Total Ignorance & \\
\hline 0 - 5 Years & $54.5 \%$ & $39.4 \%$ & $6.1 \%$ & $100.0 \%$ \\
\hline $6-11$ Years & $57.1 \%$ & $42.9 \%$ & $0.0 \%$ & $100.0 \%$ \\
\hline 12 - 17 Years & $62.5 \%$ & $31.2 \%$ & $6.2 \%$ & $100.0 \%$ \\
\hline More than 18 Years & $62.5 \%$ & $25.0 \%$ & $12.5 \%$ & $100.0 \%$ \\
\hline Total & $56.4 \%$ & $38.8 \%$ & $4.8 \%$ & $100.0 \%$ \\
\hline
\end{tabular}

Table 8. Relationship between longevity at service and knowledge/attitude relative to cultural re-patterning during transcultural client care.

\begin{tabular}{|c|c|c|c|c|}
\hline \multirow{2}{*}{ Longevity at Service } & \multicolumn{3}{|c|}{ Cultural Re-Patterning } & \multirow{2}{*}{ Total } \\
\hline & Exact & Approximate & Total Ignorance & \\
\hline 0-5 Years & $55.6 \%$ & $39.4 \%$ & $5.1 \%$ & $100.0 \%$ \\
\hline 6-11 Years & $52.4 \%$ & $45.2 \%$ & $2.4 \%$ & $100.0 \%$ \\
\hline 12-17 Years & $43.8 \%$ & $56.2 \%$ & $0.0 \%$ & $100.0 \%$ \\
\hline More than 18 Years & $50.0 \%$ & $50.0 \%$ & $0.0 \%$ & $100.0 \%$ \\
\hline Total & $53.3 \%$ & $43.0 \%$ & $3.6 \%$ & $100.0 \%$ \\
\hline
\end{tabular}

The drop at the level of "others" can be justified by the fact that, "others" is a non-homogenous mix of different categories of nurses and midwives who do not find their qualification among the qualification short listed on the questionnaire. This group constitutes auxiliary nurses, higher Nursing Diploma (HND), nurse assistants, Brevet nurses and other specialized nurses. In this light therefore, their responses could not be uniform because even though they belong to the same group, they greatly differ in term of academic and professional qualifications, longevity and in-service. It can also be observed that there was a general increase in exact knowledge/ attitude as professional qualification gets higher. In terms of total ignorance, the observation was that, ignorant participants were found mostly in the areas of lower professional qualification.

\section{Conclusions}

These observations led to the conclusion that knowledge/attitude on trans-cultural client care among nurses/ midwives in health institutions in Fako Division is influenced or determined by professional qualification. The justification for this observation and conclusion is that, as professional qualification gets higher and specialized, professional education and training becomes longer and more intense. Courses such as sociology and Anthropology, Ethics, client centred and holistic care are taught more elaborately. In this light, nurses/midwives with higher and specialized qualifications generally have more knowledge and possess better attitude as far as trans-cultural patient care is concerned. Knowledge/attitude on technical aspects of trans-cultural client care such as cultural preservation, negotiation and re-patterning are therefore influenced by the professional qualification of the nurse/midwife.

However, from a general point of view, nurses/midwives in health care institution in Fako Division are generally aware of the concept and relevance of culture in client care. But their knowledge and attitude patterning to trans-cultural patient care is insufficient as seen and justified by the variations in their academic and professional qualification and length or duration of field experience and in-service work.

Based on and with reference to the observations and the significance of the observations made or obtained from results and/or findings of the study so far, it can therefore be concluded:

- Academic/Professional qualification influence trans-cultural client care among nurses/midwives in Health 
Care Institutions in Fako Division.

- Professional profile influences trans-cultural client care among nurses/midwives in Health Care Institutions in Fako Division.

\section{Recommendations}

1) Education on trans-cultural client care be provided at level of all hospitals using weekly clinical meetings

2) Seminars be organized for all health care providers in all health facilities by regional seminars.

3) Aspects of trans-cultural client care be sourced from specialized authors and included in in-services packages for training.

\section{References}

[1] Papadopuolous, I., Tilki, M. and Taylor, G. (1998) Transcultural Care: A Guide for Health Care Professionals. Quay Books, Wilts.

[2] Kozier, B., Erb, G., Berman, A. and Snyder, S. (2004) Fundamentals of Nursing: Concepts, Process, and Practice. Pearson Education South Asia Pte Ltd., Philippines.

[3] Leininger, M.M. (1991) Culture Care Diversity and Universality: A Theory of Nursing. In: Nursing Theories: The Base for Professional Nursing Practice, Appleton and Lange, Norwalk.

[4] Leininger, M.M. (1978) Transcultural Nursing: Theories, Research, and Practice. 2nd Edition, John Wiley \& Sons, Ltd., New York.

[5] Sager, P.L. (2012) Transcultural Nursing Theories and Models. Springer Publishing Company, New York.

[6] Watson, J. (1979) Nursing: The Philosophy and Science of Caring. In: Nursing Theories: The Base for Professional Nursing Practice, Appleton and Lange, Norwalk. http://dx.doi.org/10.1097/00006216-197900340-00010

[7] Giger, J.N. and Davidhizar, R.E. (2008) Transcultural Nursing: Assessment and Intervention. 5th Edition. Mosby, London.

[8] American Association of Colleges of Nursing (2008) Tool Kit of Resources for Cultural Competent Education for Baccalaureate Nurses.

[9] Hayrol, A., Mohamed, S. and Jegak, U. (2010) The Influence of Socio-Demographic Factors on Work Performance Among Employees of Government Agriculture Agencies in Malaysia. The Journal of International Social Research, $\mathbf{3}$, 459-469.

[10] Babbie, E. (2001) The Practice of Social Research. 9th Edition. Wadsworth Thomson, Belmont.

[11] Michael, Q.P. and Michael, C. (2002) A Guide to Using Qualitative Research Methodology. Medecins sans Frontieres, London.

[12] Lagares, P.B. and Justo, P.A. (2001) Population and Sample. Sampling Techniques. DE-COMENIUS-C21, UK.

[13] Eng, J. (2003) Sample Size Estimation: How Many Individuals Should Be Studied? Radiology, 227, 209-213. http://dx.doi.org/10.1148/radiol.2272012051

[14] CliffsNotes: Orientation and Training Programs. 2014. 